\title{
Rearranging transfinite series of ordinals
}

\section{Péter Komjath}

A new, simplified proof is given of a theorem of J.L. Hickman (to be published; see also J. London Math. Soc. (2) 9 (1974), 239-244), giving an upper bound for the sums of a well-ordered series of ordinals under permutation.

In this paper we consider the following problem: let $s=\left\langle s_{\beta}: \beta\langle\alpha\rangle\right.$ be a sequence of type $\alpha$ of ordinals where $\alpha$ is an ordinal. How many distinct sums can we obtain by permuting the members of the series? To put it in a more precise form let $\Pi(\alpha)$ be the set of all permutations of $\alpha, \Sigma(\pi)=\Sigma\left(s_{\pi(\beta)}: \beta\langle\alpha\rangle\right.$ for $\pi \in \Pi(\alpha)$, $S(s)=\{\Sigma(\pi): \pi \in \Pi(\alpha)\}$, and $\sigma(s)=|S(s)|$. Given $s$ we want to get upper estimates for $\sigma(s)$. An old theorem of Sierpiniski [4] states that $\sigma(s)<\omega$ provided $\operatorname{dom}(s)=\alpha=\omega$. A more recent result of Hickman [2, 3] gives the following upper estimate

$$
\sigma(s) \leq|\alpha|
$$

for an arbitrary sequence of ordinals $s$ with $\operatorname{dom}(s)=\alpha \geq \omega$.

Our aim is to give a new and considerabiy simpler proof of Hickman's Theorem.

Our notation is standard. Lower case Latin and Greek letters denote ordinals. $\alpha+\beta, \alpha . \beta, \alpha^{\beta}$ denote ordinal operations; thus $\alpha^{\beta+1}=\alpha^{\beta} \cdot \alpha, \alpha^{\sup A}=\sup \left\{\alpha^{\gamma}: \gamma \in A\right\}$.

Received 30 November 1976. Communicated by J.L. Hickman. 
First we formulate some preliminary statements.

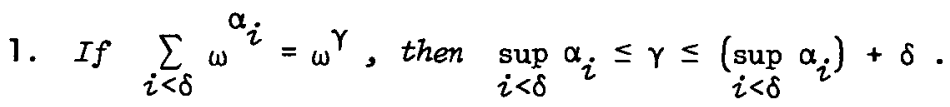

Proof. Immediate.

2. Assume $\kappa \geq \delta$ a cardinal, $F$ is a set of ordinals, $|F| \leq \kappa$, $\alpha<\mathrm{K}^{+}$. Let $F_{0}=F \cup\{x: x$ is a limit point of $F\}$ and

$$
F_{1}=\left\{x+\delta: x \in F_{0}, \delta \leq \alpha\right\}
$$

Then $\left|F_{I}\right| \leq \kappa$, and, moreover, if $\sum_{i<\delta} \omega^{\alpha_{i}}=\omega^{c}$ where $\alpha_{i} \in F, \delta \leq \alpha$, then $c \in F_{1}$.

Proof. Straightforward.

3. If $\sum_{i<\delta} \omega^{\alpha_{i}}=\omega^{c_{1}}+\ldots+\omega^{c_{n}}$ where $c_{1} \geq \ldots \geq c_{n} \quad(n<\omega)$ then there exists a $\delta_{1} \leq \delta$ such that

$$
\sum_{i<\delta} \omega^{\alpha_{i}}=\omega^{c_{1}}
$$

Proof. Put $\delta_{1}=\min \left\{\gamma: \sum_{i<\gamma} \omega^{\alpha_{i}} \geq \omega^{c_{1}}\right\}$. If $\sum_{i<\delta_{1}} \omega^{\alpha_{i}} \geq \omega^{c_{1}}+1$, the $\omega^{c_{1}}$-th member of the sum is in some $\omega^{\alpha_{i}}$. So $\sum_{i \leq i_{0}} \omega^{\alpha_{i}} \geq \omega^{c_{I}}+1$, and $\sum_{i<i_{0}} \omega^{\alpha}=\beta<\omega^{c_{1}}$ and $\delta_{1}=i_{0}+1$. But

$$
\omega^{\alpha_{0}} \leq \sum_{i<\delta} \omega^{\alpha_{i}} \leq n \cdot \omega^{c_{1}}<\omega^{c_{1}^{+1}}
$$

so $\alpha_{i_{0}} \leq c_{1}$. As $\omega^{c_{1}}$ is additively indecomposable, $\alpha_{i_{0}}=c_{1}$, and $\sum_{i \leq i_{0}} \omega^{\alpha_{i}}=\beta+\omega^{c_{1}}=\omega^{c_{1}}$. // 


$$
\begin{aligned}
& \text { 4. If } \sum_{i<\delta} \omega^{\alpha_{i}}=\omega^{c_{1}}+\ldots+\omega^{c}\left(c_{1} \geq \ldots \geq c_{n}\right) \text { then there are } \\
& 0=\delta_{0}<\delta_{1}<\ldots<\delta_{n}=\delta \text {, such that } \\
& \delta_{t} \sum_{i<\delta_{t+1}} \omega^{\alpha_{i}}=\omega^{c}{ }^{c+1} \quad(t=0,1, \ldots, n-1) .
\end{aligned}
$$

Proof. By iterating 3.

THEOREM (Hickman [3]). Assume $X$ is a set of ordinals, $\alpha$ is an ordinal, $|X| \leq \kappa$ and $\alpha<\kappa^{+}$. Then those $\alpha$-series formed from members of $X$ give at most $\kappa$ distinct sums. (Multiple occurrence is admitted.)

Proof. We may assume that every element of $X$ is a power of $\omega$. As a well-known matter of fact, every ordinal is a finite sum of powers of $\omega$. Hence if we prove the theorem for sums of lengths less than or equal to $\alpha . \omega$ and $X$ having $\omega^{x}$-type members only, then the general statement follows for sums of length $\alpha$. So let $X=\left\{\omega^{x}: x \in F\right\}$. Then by 2 and 4 the cardinality of $\left\{\sum_{i<\alpha} \omega^{\alpha_{i}}: \alpha_{i} \in F\right\}$ is at most as large as the set of finite subsets of $F_{1}$, which is less than or equal to $k$.

\section{References}

[1] S. Ginsburg, "On the distinct sums of $\lambda$-type transfinite series obtained by permuting the elements of a fixed $\lambda$-type series", Fund. Math. 39 (1952), 131-132.

[2] J.L. Hickman, "Concerning the number of sums obtainable from a countable series of ordinals by permutations that preserve the order-type", J. London Math. Soc. (2) 9 (1974/1975), 239-244.

[3] J.L. Hickman, "Some results on series of ordinals", 2. math. Logik Grundlagen Math. (to appear).

[4] Waclaw Sierpiński, "Sur les séries infinies de nombres ordinaux", Fund. Math. 36 (1949), 248-253.

Department of Algebra and Number Theory, Eötvös Lorand University, Budapest, Hungary. 